\title{
Empleo de la ultrafiltración extracorpórea aislada para el tratamiento de la insuficiencia cardiaca descompensada: dificultades técnicas y evolución clínica
}

\author{
José Luis Cobo Sánchez, Magdalena Gándara Revuelta, Raquel Pelayo Alonso, Araceli Sáenz de Buruaga Perea, \\ María Yolanda Vicente Jiménez, Marina Rojo Tordable, Carmen Higuera Roldán, Violeta Olalla Antolín
}

Diplomados en enfermería. Servicio de Nefrología. Hospital Universitario Marqués de Valdecilla. Santander

\section{Resumen}

La ultrafiltración extracorpórea aislada se ha revelado como una posible estrategia terapéutica para pacientes con insuficiencia cardíaca descompensada resistente a diuréticos. Nuestro objetivo fue evaluar la evolución clínica a corto-medio plazo de los pacientes sometidos a ultrafiltración extracorpórea aislada y las incidencias técnicas/clínicas.

Material y método: Estudio descriptivo transversal prospectivo en pacientes con insuficiencia cardíaca en estadio III-IV de la New York Heart Association resistentes al tratamiento diurético. La ultrafiltración extracorpórea aislada se realizó con el sistema Dedyca $^{\circledR}$, usando como acceso vascular un catéter venoso central. La tasa de ultrafiltración se iba ajustando en función de la tensión arterial del paciente. Para evaluar la evolución clínica se realizaron controles clínicos y analíticos antes y 1 mes después de las sesiones de ultrafiltración extracorpórea aislada. Para evaluar las complicaciones técnicas/ clínicas se revisaron los registros de enfermería.

Resultados: Se estudiaros 6 pacientes, que realizaron una media de 2 sesiones, con una duración media de 5 horas y una ultrafiltración media de $2100 c c$ por sesión. Respecto a las complicaciones técnicas, sólo un paciente presentó coagulación parcial del

Correspondencia:

Raquel Pelayo Alonso

Unidad de Hemodiálisis. Servicio de Nefrología. Hospital Universitario Marqués de Valdecilla.

Avda. Marqués de Valdecilla s/n 39008 Santander

E-mail: sanesteban6@gmail.com sistema. Ningún paciente presentó efectos adversos durante la ultrafiltración extracorpórea aislada.

Conclusiones: Tras la ultrafiltración extracorpórea aislada, aumenta la diuresis con la misma dosis de diurético e incluso con disminución progresiva. Es una técnica sencilla, sin complicaciones técnicas para enfermeras familiarizadas con técnicas de depuración extracorpórea.

PALABRAS CLAVE:

- INSUFICIENCIA CARDÍACA DESCOMPENSADA

- ULTRAFILTRACIÓN EXTRACORPÓREA

- RESISTENCIA A DIURÉTICOS

- SÍNDROME CARDIORRENAL

- ATENCIÓN DE ENFERMERÍA

Use of isolated extracorporeal ultrafiltration to treat decompensated heart failure: technical difficulties and clinical evolution

\section{Abstract}

Isolated extracorporeal ultrafiltration has shown itself to be a possible therapeutic strategy for patients with diuretic-resistant decompensated heart failure. Our aim was to assess the short-term clinical evolution of patients undergoing isolated extracorporeal ultrafiltration and the technical/clinical incidents.

Material and method: A transversal prospective descriptive study in patients with heart failure at stage III-IV according to the New York Heart Association, resistant to diuretic treatment. Isolated extracorporeal ultrafiltration was carried out with the Dedyca ${ }^{\circledR}$ system, using a central venous cathe- 
ter for vascular access. The ultrafiltration rate was adjusted in line with the patient's blood pressure. To assess the clinical evolution, clinical and analytical controls were carried out before and 1 month after the isolated extracorporeal ultrafiltration sessions. To assess the technical/clinical complications, the nursing records were reviewed.

Results: Six patients were studied, who underwent an average of 2 sessions with a mean duration of 5 hours and with a mean ultrafiltration of $2100 \mathrm{cc}$ per session. As far as technical complications are concerned, only one patient presented partial coagulation of the system. No patients presented adverse effects during the isolated extracorporeal ultrafiltration.

Conclusions: After the isolated extracorporeal ultrafiltration, diuresis increases with the same dose of diuretic and even with a progressive reduction. It is a simple technique, without technical complications for nurses who are familiar with extracorporeal purification techniques.

\section{KEY WORDS:}

- DECOMPENSATED HEART FAILURE

- EXTRACORPOREAL ULTRAFILTRATION

- RESISTANCE TO DIURETICS

- CARDIORENAL SYNDROME, NURSING CARE

\section{Introducción}

En Estados Unidos cerca de 5 millones de pacientes presentan insuficiencia cardiaca crónica, con una incidencia de 550.000 nuevos casos por año ${ }^{1}$. La insuficiencia cardiaca descompensada (ICD), sobre todo en estadíos avanzados (New York Heart Association III-IV), genera un incremento de los ingresos y costes hospitalarios ${ }^{1}$. No hay datos poblacionales de la incidencia de la insuficiencia cardiaca en España. La hospitalización por insuficiencia cardiaca está asociada con un $30 \%$ de readmisiones y un $10 \%$ de mortalidad tras los 60-90 días tras el alta hospitalaria ${ }^{2}$. Estos datos dan cuenta de la carga epidemiológica y económica que supone para el sistema sanitario la ICD.

La mayoría de los pacientes hospitalizados por ICD presentan manifestaciones clínicas de sobrecarga de volumen de líquido extracelular, como disnea, ganancia de peso, distensión yugular venosa y edema a nivel pulmonar y de las extremidades inferiores ${ }^{3}$. Las guías de práctica clínica recomiendan el uso de diuréticos como primera línea de tratamiento en la ICD para mejorar la congestión cardiaca ${ }^{4}$. Aproximadamente un 30\% de estos pacientes no responden al tratamiento con diuréticos ${ }^{5}$. Aunque los diuréticos mejoran la sobrecarga de volumen, su uso está relacionado con un incremento de la mortalidad ${ }^{6-8}$, un empeoramiento de la función renal y progresión de la insuficiencia cardiaca ${ }^{9-11}$.

Laultrafiltración extracorpóreaaislada (UEA) se harevelado como una posible estrategia terapéutica en pacientes con ICD resistente a diuréticos ${ }^{12}$. Nuestro objetivo fue evaluar la evolución clínica de pacientes con ICD sometidos a UEA a corto-medio plazo; y evaluar las incidencias técnicas/clínicas de los pacientes sometidos a UEA.

\section{Material y método}

Realizamos un estudio descriptivo transversal prospectivo en pacientes con ICD tratados con UEA en nuestra unidad de hemodiálisis en el año 2011. Como criterios de inclusión en el estudio se establecieron: mayores de 18 años, insuficiencia cardiaca en estadío III-IV de la New York Heart Association (NYHA), resistentes al tratamiento diurético (diuresis $<500 \mathrm{ml} / 24 \mathrm{~h}$ con dosis máximas de furosemida hasta $250 \mathrm{mg} / 24 \mathrm{~h}$ o asociación con tiazidas 0 antialdosterónicos). Como criterios de exclusión se establecieron: hematocrito $>42 \%$, tratamiento con hemodiálisis, filtrado glomerular estimado $<30 \mathrm{ml} / \mathrm{min}$, soporte inotrópico endovenoso, tensión arterial sistólica $<80 \mathrm{mmHg}$ y alteraciones de la coagulación.

La UEA se realizó con el sistema DEDYCA ${ }^{\circledR}$ que utiliza una polisulfona de $0,25 \mathrm{~m}^{2}$ de superficie y un volumen de cebado del circuito de $100 \mathrm{ml}$. Como acceso vascular se utilizó un catéter venoso central insertado en yugular/ femoral (catéter trio de alto flujo de $13 \mathrm{~F}$ de diámetro y $20 \mathrm{~cm}$ de longitud). El flujo sanguíneo utilizado estuvo comprendido entre 50 y $100 \mathrm{ml} / \mathrm{min}$. La ultrafiltración se produce mediante la aplicación de succión por una bomba peristáltica. La tasa de ultrafiltración (TUF) se iba ajustando en función de la tensión arterial sistólica (TAS) del paciente: TAS $\leq 80 \mathrm{mmHg}$ TUF $50 \mathrm{ml} / \mathrm{h}$, TAS 80-90 $\mathrm{mmHg}$ TUF $100 \mathrm{ml} / \mathrm{h}$, TAS $91-100 \mathrm{mmHg}$ TUF $200 \mathrm{ml} / \mathrm{h}$, TAS $>100 \mathrm{mmHg}$ TUF $300-500 \mathrm{ml} / \mathrm{h}$ (según tolerancia clínica). Para evaluar la evolución clínica se realizaron controles clínicos y analíticos antes y 1 mes después de las sesiones de UEA. Los criterios de clasificación de la 
NYHA, que establece cuatro categorías en base a como la insuficiencia cardiaca afecta a la actividad física del paciente (tabla 1$)^{13}$. Para evaluar las complicaciones técnicas/clínicas se revisaron los registros de la UEA.

\begin{tabular}{|l|l|}
\hline Clase funcional & Actividad física del paciente \\
\hline Clase I & $\begin{array}{l}\text { Pacientes sin limitación de la actividad física } \\
\text { normal. }\end{array}$ \\
\hline Clase II & $\begin{array}{l}\text { Pacientes con ligera limitación de la actividad } \\
\text { física. }\end{array}$ \\
\hline Clase III & $\begin{array}{l}\text { Pacientes con acusada limitación de la activi- } \\
\text { dad física; cualquier actividad física provoca la } \\
\text { aparición de los síntomas. }\end{array}$ \\
\hline Clase IV & $\begin{array}{l}\text { Pacientes con síntomas de insuficiencia cardia- } \\
\text { ca en reposo. }\end{array}$ \\
\hline
\end{tabular}

Tabla 1. Clasificación funcional de la insuficiencia cardíaca según la New York Heart Association (NYHA).

Las variables estudiadas se analizaron mediante el paquete estadístico SPSS versión 15.0 para Windows. Los datos son presentados como frecuencias y porcentajes para las variables categóricas; o como medias, desviación típica y rangos para las variables continuas.

\section{Resultados}

Se estudiaron 6 pacientes: 50\%mujeres, con una edad media de 77,5 años (rango 62-87 años, desviación típica 8,47 años). De media se realizaron 2 sesiones (rango 2-3 sesiones), con una duración media de 5 horas y una ultrafiltración media de $2100 \mathrm{ml}$ por sesión (rango 1750-2500 ml, desviación típica $304 \mathrm{ml}$ ). Las características clínicas previas al tratamiento con UEA y al mes, se muestran en la tabla 2.
En cuanto a la dosis de diurético, pre UEA el $100 \%$ de los pacientes fueron tratados con furosemida $250 \mathrm{mg}$ e.v. $/ 24 \mathrm{~h}$ + hidroclorotiazida $50 \mathrm{mg} / 24 \mathrm{~h}$; al mes de realizar la UEA: furosemida $240 \mathrm{mg} / 24 \mathrm{~h}$ + hidroclorotiazida $50 \mathrm{mg} / 24 \mathrm{~h}$ (36\%), furosemida $160 \mathrm{mg} / 24 \mathrm{~h}+$ hidroclorotiazida $25 \mathrm{mg} / 24 \mathrm{~h}$ (16\%), furosemida $120 \mathrm{mg} / 24 \mathrm{~h}$ + hidroclorotiazida $25 \mathrm{mg} / 24 \mathrm{~h}$ (16\%), furosemida $120 \mathrm{mg} / 24 \mathrm{~h}$ (16\%) y furosemida 80 $\mathrm{mg} / 24 \mathrm{~h}(16 \%)$.

Respecto a las complicaciones técnicas, sólo un paciente presentó coagulación parcial del sistema. Ningún paciente presentó efectos adversos durante la UEA, sólo una paciente presentó hipotensión arterial sostenida, similar a su situación basal, sin repercusión clínica.

\section{Discusión}

Recientemente, se ha redefinido en una conferencia de consenso la clasificación del síndrome cardiorrenal (SCR) en cinco subtipos que reflejan la fisiopatología y el contexto temporal de la disfunción cardíaca y renal concomitantes ${ }^{14}$. En el tipo 2 de SCR, trastornos crónicos de la función cardíaca, como la insuficiencia cardíaca congestiva crónica, producen una insuficiencia renal crónica progresiva ${ }^{15}$. Este es el caso de los pacientes de nuestro estudio, en los que el tratamiento con diuréticos tiene una limitada eficacia ${ }^{16,17}$. En estos casos, las técnicas de extracción extracorpórea de fluidos se presentan como una alternativa terapéutica de rescate.

Nuestros resultados muestran una mejoría clara en cuanto a la clase funcional en este tipo de pacientes. Esta mejoría clínica a corto-medio plazo también ha

\begin{tabular}{|l|c|c|}
\hline Parámetro & Pre UEA & Post UEA \\
\hline Clase funcional NYHA & IV $(100 \%)$ & II $(66 \%)$, III $(34 \%)$ \\
\hline Tensión arterial sistólica (mmHg) & $109,67 \pm 16,03(94-137)$ & $118,33 \pm 16,69(104-143)$ \\
\hline Tensión arterial diastólica (mmHg) & $56,17 \pm 6,46(43-49)$ & $60,83 \pm 10,26(50-76)$ \\
\hline Frecuencia cardíaca (Ipm) & $65,83 \pm 4,11(61-70)$ & $69,83 \pm 6,36(60-79)$ \\
\hline Peso (kg) & $89 \pm 2,5(55-110)$ & $83 \pm 2,7(42-101)$ \\
\hline Diuresis (ml/24h) & $816,67 \pm 592,3(450-1500)$ & $145,17 \pm 37,15(95-210)$ \\
\hline Urea sérica (mg/dl) & $158 \pm 45,6(124-249)$ & $1,41 \pm 0,35(0,8-1,8)$ \\
\hline Creatinina sérica (mg/dl) & $1,65 \pm 0,27(1,2-1,9)$ & $37,83 \pm 11,89(27-60)$ \\
\hline MDRD (ml/min/1,73 m2) & $37,67 \pm 7,09(27-46)$ & $135,17 \pm 2,71(131-139)$ \\
\hline Sodio sérico (mEq/L) & $134,5 \pm 4,68(128-140)$ & $11,76 \pm 1,99(10-15,6)$ \\
\hline Hemoglobina (mg/dl) & $10,48 \pm 1,57(8,8-13)$ & $35,6 \pm 6,03(31-47)$ \\
\hline Hematocrito(\%) & $32,17 \pm 4,78(28-40)$ & $(1200-3250)$ \\
\hline
\end{tabular}

Tabla 2. Parámetros clínicos antes (pre UEA) y al mes (post UEA) de realizar las sesiones de UEA. Para las variables categóricas se muestra el porcentaje, y para las variables continuas se muestra la media \pm desviación típica y el rango. 
sido descrita en otros estudios que evaluaron la utilidad de la UEA en la ICD ${ }^{18-21}$. Costanzo et al ${ }^{18}$ estudiaron la utilidad de la UEA en pacientes con las mismas características de nuestro estudio (ICD y resistencia a diuréticos, edad media 74 años, enfermedad isquémica $75 \%$, fracción de eyección media del 3\%), a los que sometieron a 2,6 sesiones de media con una duración de 8 horas, y una ultrafiltración media total de las sesiones de $8600 \mathrm{ml}$. Observaron una mejoría clínica en los síntomas de sobrecarga hídrica (peso $p=0,006$, puntuaciones del Minessota Living with Heart Failure $p=0,0003$ y evaluación global $p=0,00003)$, tanto al alta como a los 30 y 90 días. Nalesso et a ${ }^{19}$ estudiaron 21 pacientes con ICD que se sometieron a una ultrafiltración media de $2600 \mathrm{ml}$ durante sesiones de 6,4 horas de media, consiguiendo una reducción de peso media desde $91,9 \mathrm{~kg}$ hasta $89,3 \mathrm{~kg}(p<0,0001)$, mejorando la congestión cardiaca, sin cambios en la presión arterial, frecuencia cardiaca, nivel de electrolitos o en el hematocrito. Nuestros resultados corroboran estos hallazgos. Los resultados del estudio de Constanzo ${ }^{18}$ sugieren que en pacientes con insuficiencia cardiaca con sobrecarga de volumen y resistencia a los diuréticos, la ultrafiltración temprana antes del tratamiento con diuréticos intravenosos disminuye la longitud de la estancia y los reingresos, y se asocia con un beneficio clínico que perdura a los 90 días. En nuestro estudio no incluimos la variable de a qué días tras la UEA los pacientes fueron dados de alta, ya que la causa principal del ingreso de varios de ellos no fue un episodio de descompensación de la insuficiencia cardiaca. En los casos en los que la causa principal de ingreso fue la ICD, los pacientes fueron dados de alta a los 3 días tras las sesiones de UEA, lo que disminuyó los costes de la misma. El ensayo clínico UNLOAD ${ }^{20}$, que comparaba la ultrafiltración veno-venosa con el uso de diuréticos endovenosos en 200 pacientes con ICD, también observó una mayor mejoría clínica, menor estancia hospitalaria y mejores resultados clínicos a corto-medio plazo en el grupo que se sometió a la ultrafiltración.

Bart et a ${ }^{21}$ compararon los resultados clínicos en 20 pacientes con ICD con tratamiento diurético endovenoso con otros 20 pacientes con ICD tratados con UEA en sesiones de 8 horas, consiguiendo mayor cantidad de volumen extraído con la UEA ( $2838 \mathrm{ml}$ versus $4650 \mathrm{ml}$; $p=0,001$ ). Los resultados de este estudio, al igual que los del UNLOAD, demuestran que la estrategia con UEA obtiene mejores resultados clínicos que el tratamiento con diuréticos endovenosos.
Nuestros resultados muestran un aumento de la diuresis con igual o menor dosis de diurético tras el tratamiento con UEA, manteniendo una función renal y un hematocrito estable, con ligera corrección de la hiponatremia. Estos resultados coinciden con los hallados en la literatura ${ }^{20-22}$. En nuestro estudio no hemos podido evaluar la evolución del péptido natriurético tipo $B$, al no disponer de dicha determinación en nuestro hospital. De entre los biomarcadores cardiacos, la determinación plasmática de este péptido y del fragmento aminoterminal de la proteína precursora en el plasma han mostrado ser especialmente útiles para establecer el diagnóstico y pronóstico de la ICD ${ }^{23}$.

Respecto a las incidencias técnicas y a la tolerancia clínica de la UEA, en nuestro estudio estos efectos adversos fueron anecdóticos, probablemente derivado porque el manejo de la UEA fue realizado por enfermeras nefrológicas experimentadas, acostumbradas al manejo de terapias de depuración extracorpórea. Se han descrito problemas relacionados con el acceso vascular y con complicaciones con el sistema, como infección, trombosis, embolismo aéreo, o hemorragia debido a la desconexión del retorno venoso del catéter ${ }^{24}$. En nuestra opinión, este tipo de técnicas han de ser realizadas por enfermeras con conocimientos y habilidades en este tipo de terapias (cualidades que reúne la enfermería nefrológica), lo que repercute en una mayor seguridad del paciente y en el éxito de un programa de UEA como tratamiento de la ICD. Además de en la aplicación de técnicas, la enfermera tiene un papel decisivo en una unidad de insuficiencia cardiaca, aportando una visión integral de este tipo de pacientes, haciendo especial hincapié en el autocuidado y en el manejo de complicaciones durante los episodios agudos 25,26 .

\section{Conclusiones}

La UEA mejora de forma evidente la clase funcional, pasando de un estadío IV a un estadío II. A corto plazo se objetiva un aumento de la diuresis con la misma dosis de diurético e incluso con disminución progresiva. No se producen alteraciones analíticas, con estabilidad de la función renal y hematocrito, con ligera corrección de la hiponatremia. La UEA es un método sencillo, sin complicaciones técnicas para enfermeras familiarizadas con el manejo de técnicas de depuración extracorpórea. La UEA es bien tolerada por los pacientes con ICD. 
Recibido: 10 Noviembre 2013

Revisado: 20 Noviembre 2013

Modificado: 20 Noviembre 2013

Aceptado: 22 Noviembre 2013

\section{Bibliografía}

1. Roger VL, Go AS, Lloyd-Jones DM, Adams RJ, Berry JD, Brown TM, et al. Heart disease and stroke statistics - 2011 update: A report from the American Heart Association. Circulation 2011; 123: e18 - e209.

2. Fonarow GC, Abraham WT, Albert NM, Stough WG, Gheorghiade M, Greenberg BH, et al. Factors identified as precipitating hospital admissions for heart failure and clinical outcomes: Findings from OPTIMIZE-HF. Arch Intern Med 2008; 168: 847 $-854$.

3. Adams KF Jr, Fonarow GC, Emerman $\mathrm{CL}$, et al. Characteristics and outcomes of patients hospitalized for heart failure in the United States: rationale, design, and preliminary observations from the first 100,000 cases in the Acute Decompensated Heart Failure National Registry (ADHERE). Am Heart J. 2005;149(2):209-216.

4. Jessup M, Abraham WT, Casey DE, et al. 2009 Focused update: ACCF/AHA Guidelines for the Diagnosis and Management of Heart Failure in Adults: a report of the American College of Cardiology Foundation/American Heart Association Task Force on Practice Guidelines: developed in collaboration with the International Society for Heart and Lung Transplantation. Circulation 2009;119(14):19772016.

5. Fonarow GC. The Acute Decompensated Heart Failure National Registry (ADHERE): opportunities to improve care of patients hospitalized with acute decompensated heart failure. Rev Cardiovasc Med. 2003;4(suppl 7):S21-S30.

6. Peacock WF, Costanzo MR, De Marco T, et al. Impact of intravenous loop diuretics on outcomes of patients hospitalized with acute decompensated heart failure: insights from the ADHERE registry. Cardiology. 2009;113(1):12-19.
7. Harjai KJ, Dinshaw HK, Nunez E, et al. The prognostic implications of outpatient diuretic dose in heart failure. Int J Cardiol.1999;71(3):219-225.

8. Domanski $M$, Norman J, Pitt $B$, Haigney $M$, Hanlon $\mathrm{S}$, Peyster E. Diuretic use, progressive heart failure, and death in patients in the Studies Of Left Ventricular Dysfunction (SOLVD). J Am Coll Cardiol. 2003;42(4):705-708.

9. Francis GS, Siegel RM, Goldsmith SR, Olivari MT, Levine TB, Cohn JN. Acute vasoconstrictor response to intravenous furosemide in patients with chronic congestive heart failure. Activation of the neurohumoral axis. Ann Intern Med. 1985;103(1): 1-6.

10. Gottlieb SS, Skettino SL, Wolff A, et al. Effects of BG9719 (CVT-124), an Al-adenosine receptor antagonist, and furosemide on glomerular filtration rate and natriuresis in patients with congestive heart failure. J Am Coll Cardiol. 2000;35(1):56-59.

11. Schrier RW. Role of diminished renal function in cardiovascular mortality: marker or pathogenetic factor? J Am Coll Cardiol. 2006;47(1):1-8.

12. Dahle TG, Sobotka PA, Boyle AJ. A practical guide for ultrafiltration in acute decompensated heart failure. Congest Heart Fail. 2008;14(2):83-88.

13. Aldámiz-Echevarría Iraurgui B. Guía de práctica clínica: Insuficienciencia cardiaca. Fisterra. [Consultado el 10 de Octubre de 2011] Disponible en: http://www.fisterra.com/guias-clinicas/insuficiencia-cardiaca/.

14. Ronco C, McCullough P, Anker SD. Cardio-renal syndromes: report from the consensus conference of the acute dialysis quality initiative. Eur Heart $\mathrm{J}$ 2010;31:703-11.

15. Barrio Lucía V, Fernández Juárez G. Trastornos del agua en el síndrome cardiorrenal. Nefrología Sup Ext 2011;2(6):29-34.

16. Adams KF, Fonarow GC, Emerman CL, et al. Characteristics and outcomes of patients hospitalized for heart failure in the United States: rationale, design, and preliminary observations from the first 100,000 cases in the Acute Decompensated Heart Failure National Registry (ADHERE). Am Heart J. 2005;149:209-16. 
17. Felker GM, Lee $K L$, Bull DA, et al. Diuretic strategies evaluation in patients with acute decompensated heart failure. N Engl J Med. 2011;364:797-805.

18. Costanzo MR, Saltzberg M, $0^{\prime}$ Sullivan J, Sobotka P. Early ultrafiltration in patients with decompensated heart failure and diuretic resistance. J Am Coll Cardiol. 2005;46(11):2047-51.

19. Nalesso F, Garzotto F, Ronco C. Technical aspects of extracorporeal ultrafiltration: mechanisms, monitoring and dedicated technology. Contrib $\mathrm{Ne}$ phrol. 2010;164:199-208.

20. Costanzo MR, Saltzberg MT, Jessup M, et al. UItrafiltration is associated with fewer re-hospitalizations than continuous diuretic infusion in patients with decompensated heart failure: results from UNLOAD. J Cardiac Fail. 2010;16:277-84.

21. Bart BA, Boyle A, Bank AJ, et al. Randomized controlled trial of ultrafiltration versus usual care for hospitalized patients with heart failure: relief for acutely fluid overloaded patients with decompensated congestive heart failure. J Am Coll Cardiol. 2005;46:2043-6.
22. Rogers HL, Marshall J, Bock J, Dowling TC, Feller $E$, Robinson $S$, et al. A randomized, controlled trial of the renal effects of ultrafiltration as compared to furosemide in patients with acute decompensated heart failure. J Card Fail. 2008;14:1-5.

23. Baggish $A L$, Van Kimmenade RR, Januzzi Jr JL. Amino-terminal pro-B-type natriuretic peptide testing and prognosis in patients with acute dyspnea, including those with acute heart failure. Am J Cardiol. 2008;101:49-55.

24. Shin JT, Dec GW. Ultrafiltration should not replace diuretics for the initial treatment of acute decompensated heart failure. Circ Heart Fail. 2009;2:505-511.

25. Albert NM. Fluid management strategies in heart failure. Crit Care Nurse. 2012 Apr;32(2):20-32.

26. Nieto Montesinos MJ, García Fernández $Y$, Atrio Padrón ML. Plan de cuidados a pacientes con insuficiencia cardíaca congestiva (I.C.C.). Rev Enferm Cardiol 2004; 31:23-25. 